3. Christen U, Von Herrath MG. Infections and autoimmunity-good or bad? J Immunol 2005 ; $174: 7481-6$

4. Soulas P, Woods A, Jaulhac B, et al. Autoantigen, innate immunity, and $T$ cells cooperate to break $B$ cell tolerance during bacterial infection.

J Clin Invest 2005 ; 115 : 2257-67.

5. Akira S, Takeda K. Toll-like receptor signalling. Nat Rev 2004 ; 4 : 499-511.
6. Alexopoulou L, Thomas V, Schnare M, et al. Hyporesponsiveness to vaccination with Borrelia burgdorferi OspA in humans and in TLR1- and TLR2deficient mice. Nat Med 2002; $8: 878-84$.

7. Julien S, Soulas P, Garaud JC, et al. B cell positive selection by soluble self-antigen. J Immunol 2002 ; $169:$ 4198-204

8. Dorner T, Egerer K, Feist $\varepsilon$, Burmester GR. Rheumatoid factor revisited. Curr Opin Rheumatol 2004 ; 16: 246-53.
9. Rui L, Vinuesa CG, Blasioli J, Goodnow CC. Resistance to CpG DNA-induced autoimmunity through tolerogenic $B$ cell antigen receptor ERK signaling. Nat Immunol 2003; $4: 594-600$.

10. Roosnek $\varepsilon$, Lanzavecchia, A. Efficient and selective presentation of antigen-antibody complexes by rheumatoid factor B cells. J Exp Med 1991 ; 173 : 487-9.

\title{
NOUVELLE
}

\section{Réponse coordonnée des voies de surveillance de l'intégrité du génome au stress réplicatif chez Schizosaccharomyces pombe}

Stefania Francesconi

L'intégrité du génome des organismes vivants est constamment menacée par différents facteurs extrinsèques ou intrinsèques aux cellules. Pour faire face à cette constante menace, des systèmes de surveillance (checkpoints) contrôlent l'accomplissement de la réplication de I'ADN et la présence de lésions sur celuici. L'activation de ces voies aboutit à l'arrêt du cycle cellulaire en liaison avec l'induction de la réparation de l'ADN, I'inhibition de la réplication et l'activation des systèmes de récupération [ 1 , 2]. En outre, le système de surveillance du fuseau mitotique assure à chaque cycle cellulaire la ségrégation correcte des chromatides sœurs dans les cellules filles [3]. II faut souligner que les voies de surveillance de l'intégrité du génome sont conservées des levures à l'homme. Les cassures double-brin (CDB) de l'ADN sont parmi les lésions les plus dangereuses pour l'intégrité du génome. Elles peuvent se former spontanément au cours de la réplication de l'ADN ou être induites par des agents physiques comme les radiations ionisantes (RI), ou chimiques comme la camptothécine (CPT), un inhibiteur de la topo-isomérase I (Top I). Au cours de la réplication de l'ADN, l'inhibition de la Top I provoque l'effondrement des fourches de réplication et, par conséquent, la formation de CDB [4]. Chez la levure Schizosaccharomyces pombe et chez les vertébrés, l'exposition des cellules aux RI ou à la CPT induit l'activation de la kinase Chkl (checkpoint 1), un des effecteurs de la voie de surveillance de la réparation de l'ADN.

ChezS. pombe, l'activation de Chkl dépend de sa phosphorylation au niveau de la sérine 345 par la kinase Rad3, homologue de la kinase ATR (ataxia telangiectasia and rad3 related) des vertébrés. L'activation de Chkl par Rad3 conduit à un arrêt du cycle cellulaire en phase $\mathrm{G} 2$, et donc à un retard d'entrée en mitose [5]. Le passage en mitose sera déclenché lorsque les lésions sur l'ADN sont réparées.

Pour que Rad3 puisse phosphoryler Chk1, la présence de la protéine Crb2 est nécessaire $[6,7]$. Crb2 est une protéine contenant, dans sa partie carboxyterminale, un domaine Tudor et deux domaines BRCT (BRCAl C-terminus). Cette partie de la protéine est fortement homologue à la protéine de contrôle Rad9 de S. cerevisiae et à la
CNRS UMR2027, Institut Curie,

Centre Universitaire d'Orsay,

91405 Orsay, France.

sfrances@curie.u-psud.fr

protéine suppresseur de tumeur 53BP1 (p53 binding protein 1) humaine [8]. En fait, nous pouvons considérer la protéine Crb2 comme l'homologue fonctionnel de la 53BPl : les deux protéines sont requises pour l'activation du système de surveillance de la réparation de l'ADN en réponse aux $C D B$, et elles sont recrutées au niveau des lésions de I'ADN en fonction des modifications de la chromatine.

L'allèle mutant $\mathrm{crb2PH}$ (mutations dans la proline 629 et l'histidine 632) code une protéine dont les domaines BRCT ne sont pas fonctionnels. Nous avons montré, ainsi que d'autres équipes, que ces domaines sont indispensables à l'activation de Chkl par Rad3, et donc à l'activation du système de surveillance de la réparation de I'ADN. En effet, les cellules crb2PH sont sensibles à différents agents génotoxiques (RI par exemple), car elles sont incapables d'activer Chkl et, par conséquent, elles sont inaptes à bloquer l'entrée en mitose lorsque I'ADN est endommagé. Nous avons observé que, contrairement aux cellules qui ne possèdent pas le gène crb2, les cellules qui expriment l'allèle mutant $c r b 2 P H$ restent résistantes à des génotoxiques qui interfèrent avec la réplication de l'ADN, notamment à la CPT. Puisque les cellules $\mathrm{crb2PH}$ ne peuvent 
pas promouvoir l'activation de Chkl Rad3 dépendante, la résistance à la CPT n'est pas le résultat de l'activation du point de contrôle qui retarde l'entrée en mitose des cellules dont l'ADN contient des CDB. II fallait donc rechercher l'origine de cette résistance.

Nous avons caractérisé la résistance à la CPT des cellules crb2PH. Nous avons démontré que celle-ci dépend de la présence de la protéine Chkl, mais pas de son activation par la kinase Rad3. En effet, un allèle qui exprime une protéine Chkl mutée au niveau de la sérine 345 (chk1S345A) est aussi résistant à la CPT que les cellules $\mathrm{crb2} P H$, bien que la voie de surveillance de l'entrée en mitose ne soit pas fonctionnelle. De plus, la résistance à la CPT des cellules $\mathrm{crb} 2 \mathrm{PH}$ et chk1S345A est liée à la présence de la protéine Mad2 qui intervient dans la voie de contrôle du fuseau mitotique. Cette autre voie empêche les cellules qui se trouvent en métaphase de passer en anaphase dans le cas où les chromosomes dupliqués ne sont pas correctement attachés au fuseau mitotique. Dès que les microtubules ont amarré convenablement tous les chromosomes, le contrôle s'éteint et la mitose se poursuit jusqu'à son terme. C'est de cette manière que la répartition correcte des chromosomes est assurée dans les cellules filles. Nous avons démontré que Crb2, Chkl et Mad2 agissent dans la même voie qui permet aux cellules mutées au point de contrôle de l'entrée en mitose de survivre à la présence de $C D B$ engendrés au cours de la réplication de l'ADN.

Grâce à l'utilisation d'une protéine fluorescente qui se localise dans les corps basaux de la cellule, nous avons suivi la progression de la mitose dans les cellules crb2PH exposées à la CPT. Nous avons ainsi pu observer que le traitement par la CPT des cellules crb2PH provoque un retard de sortie de la mitose. Autre-

Taux de perte du chromosome

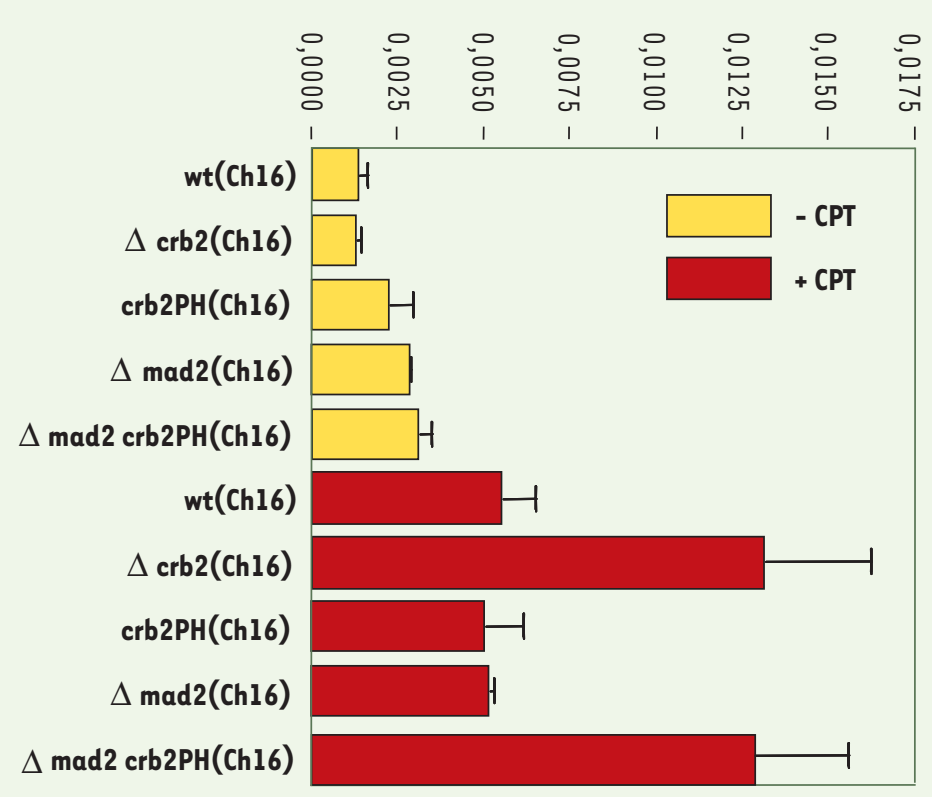

Figure 1. Étabissement du taux de perte du minichromosome 16 (Ch16) dans les différentes souches. Les cellules crb2PH traitées ou non par la CPT ont un taux similaire à celui des cellules sauvages lorsque la protéine Mad2 est présente, alors que le double mutant crb2PH contenant la délétion de mad2 ( $\mathrm{crb2PH} \Delta \operatorname{mad} 2$ ) a un taux de perte du minichromosome similaire à celui de la souche qui porte la délétion de crb2 ( $\Delta c r b 2)$. ment dit, les cellules doublent le temps qu'elles passent en métaphase. Cela est vrai aussi pour les cellules chk1S345A traitées à la CPT. Ce retard de sortie de la mitose dépend de la présence des protéines Chk1 et Mad2, alors qu'il ne dépend pas de la phosphorylation de Chkl par Rad3.

La voie Crb2/Chkl est donc capable de maintenir la voie de surveillance du fuseau mitotique activé, pour un temps plus long lorsque les cellules entrent en mitose avec des $C D B$ engendrés par un stress réplicatif. Cette fonction est nécessaire et suffisante à la survie cellulaire, et surtout au maintien de l'intégrité du génome des cellules mutées dans le système de surveillance d'entrée en mitose activé par des lésions de l'ADN. $\varepsilon$ n effet, les cellules crb2PH traitées par la CPT ont une stabilité chromosomique similaire à celle des cellules sauvages, et cette stabilité nécessite la protéine Mad2 (Figure 1).

Ces observations permettent de proposer un modèle (Figure 2) selon lequel la voie Crb2/Chkl agirait sur deux étapes du cycle cellulaire lorsque les cellules ont subi un stress réplicatif: l'entrée en mitose et la sortie de la mitose. La première étape est assurée par l'activation de Chkl Rad3 dépendante grâce à la présence des domaines BRCT de la protéine Crb2. La deuxième étape est assurée par la partie aminoterminale de Crb2 qui, via Chkl, agit sur le système de surveillance du fuseau mitotique. Les événements moléculaires de cette deuxième étape restent encore à déterminer.

Comme chez S. pombe, les cellules humaines activent la kinase Chkl en réponse au traitement par la CPT. Comprendre la réponse cellulaire à cette molécule est d'autant plus important qu'elle est couramment utilisée en chimiothérapie. Les cellules tumorales sont souvent mutées dans les systèmes de surveillance, et elles sont donc très vulnérables aux agents qui endommagent I'ADN. Cependant, il existe des cancers résistant à la CPT. 
Des mécanismes multiples et différents peuvent expliquer cette résistance, de l'insuffisante accumulation de la drogue à des modifications de la réponse cellulaire à l'inhibition de la Top I. Notre travail suggère la possibilité que des cellules déficientes au point de contrôle d'entrée en mitose

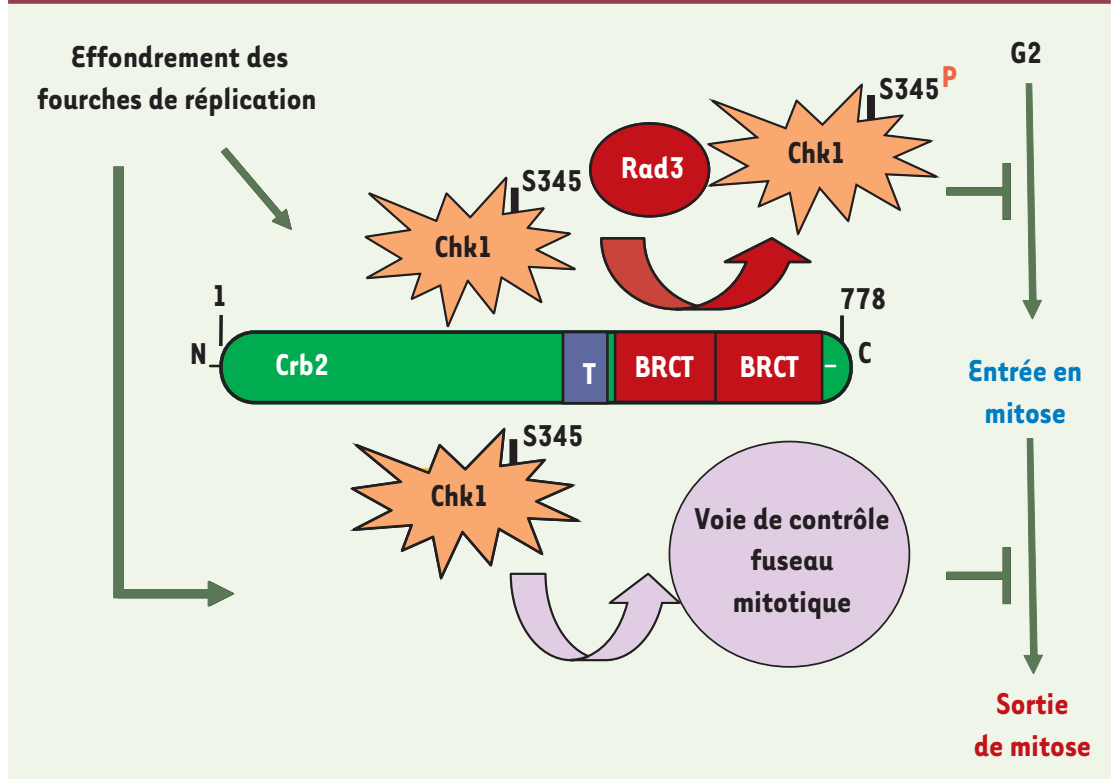

Figure 2. Séparation des fonctions de la voie de surveillance aboutissant à la kinase Chk1 grâce à l'allèle crb2PH. Lorsque des cellules subissent un stress réplicatif, cette voie bloque l'entrée en mitose des cellules par activation de Rad3 dépendante de Chk1. Pour cela, les domaines BRCT de la protéine Crb2 sont essentiels. En même temps, la voie bloque la sortie de la mitose en agissant sur la voie de surveillance du fuseau mitotique. Pour cela, la partie aminoterminale de la protéine Crb2 est nécessaire. Cette deuxième fonction assure le maintien de l'intégrité du génome des cellules qui entrent en mitose avec des CDB engendrées au cours de la réplication de l'ADN. puissent survivre à la CPT grâce à un allongement du temps d'activation du système de surveillance du fuseau mitotique. $\diamond$

Repair of and checkpoint response to topoisomerase I-mediated DNA damage in Schizosaccharomyces pombe

\section{RéFÉRENCES}

1. Carr AM. DNA structure dependent checkpoints as regulators of DNA repair. DNA Repair 2002 ; 1: 983-94.

2. McGowan $\mathrm{CH}$, Russell P. The DNA damage response: sensing and signaling. Curr Opin Cell Biol 2004 $16: 629-33$.

3. Bharadwaj R, $Y u H$. The spindle checkpoint, aneuploidy, and cancer. Oncogene 2004 ; $23: 2016-27$

4. Pommier Y, Redon C, Rao VA, et al. Repair of and checkpoint response to topoisomerase I-mediated DNA damage. Mutat Res 2003 ; 532 : 173-203.

5. Capasso H, Palermo C, Wan S, et al. Phosphorylation activates Chkl and is required for checkpointmediated cell cycle arrest. J Cell Sci 2002 ; $115: 4555-64$.

6. Saka Y, Esashi F, Matsusaka T, et al. Damage and replication checkpoint control in fission yeast is ensured by interactions of Crb2, a protein with BRCT motif, with Cut5 and Chk1. Genes Dev 1997 ; 11: 3387-400.

7. Willson J, Wilson S, Warr N, Watts FZ. Isolation and characterization of the Schizosaccharomyces pombe rhp9 gene: a gene required for the DNA damage checkpoint but not the replication checkpoint. Nucleic Acids Res 1997 ; 25 : 2138-46.

8. Mochan TA, Venere M, DiTullio RA, Halazonetis TD. $53 \mathrm{BP} 1$, an activator of ATM in response to DNA damage. DNA Repair 2004 ; 3 : 945-52.

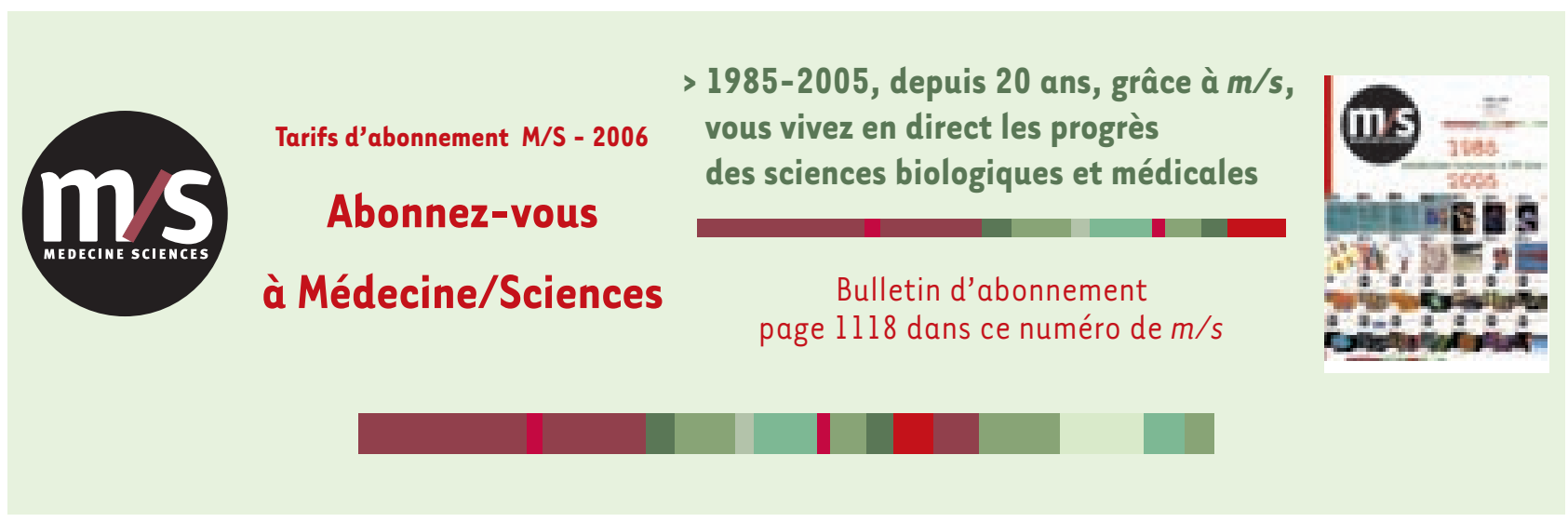

